\title{
Anchorless Underwater Acoustic Localization
}

\author{
Elizaveta Dubrovinskaya* ${ }^{*}$, Roee Diamant ${ }^{\S}$, Paolo Casari* \\ *IMDEA Networks Institute, Madrid, Spain $\quad \ddagger$ Universidad Carlos III, Madrid, Spain \\ ${ }^{\S}$ Department of Marine Technologies, University of Haifa, Israel
}

\begin{abstract}
We consider the problem of estimating the trajectory of a submerged source emitting acoustic signals without using any anchor nodes or receiving array. This approach is required for several applications, including the localization of acoustic sources such as marine mammals or underwater vehicles, for which the cost of covering a broad area with multiple receivers would be excessively high. Since multi-lateration is impossible in this scenario, we perform localization by incorporating bathymetry information. Specifically, we assume that the receiver retains knowledge of the environmental parameters that affect the signal propagation, and that the bathymetry of the area is sufficiently diverse to induce distinguishable channel impulse responses for different source locations. Our method compares the channel estimates obtained from the received acoustic signals against a database of channel responses, pre-computed through an acoustic ray tracing model. The set of possible node locations that result are then organized in trellis form to obtain a final estimate of the source's trajectory via a path tracking method similar to the Viterbi algorithm. Our results show that the proposed approach can estimate node locations and paths with very small error, provided that the receiver has sufficiently accurate and up-todate environmental information.
\end{abstract}

\section{INTRODUCTION}

Several applications in the field of ocean exploration, including, e.g., natural habitat preservation, control of secure areas, and environmental monitoring often require to estimate the location of a target emitting acoustic signals. For sporadic targets, whose appearance at a given location and time is not certain, many receivers should be deployed in order to cover a large area, and thus achieve a high probability to detect and localize the target. Example applications are the localization of marine mammals or underwater vehicles. In these cases, the required equipment is deployed sparsely and for long periods of time, in order to balance a sufficient target detection probability with long term deployment constraints. As a result, the signals are practically detected only by a single receiver. While several localization methods exist to estimate the position of a submerged device [1], most of them require the presence of several anchor nodes [2], or prescribe message exchanges between the device and the anchors [3]. Conversely, in our setting, detections are to be made by a single receiver. Because the source is unknown and is not cooperative, the effective result is that current methods would only detect the signals and avoid source localization [4].

In this paper, we extend the state of the art and propose an approach to localize a source emitting acoustic signals via a single receiver moored at a fixed location. Our approach is inspired by indoor fingerprinting localization approaches, e.g., [5], and hinges on the spatial diversity of the sea bottom bathymetry. Specifically, we target those environments where the bathymetry and the sound speed profile (SSP) in the water column induce different channel impulse responses (CIRs) for different emitter-receiver location pairs. This is often the case for shallow-water environments with a diverse non-flat bathymetry, but also for deeper waters where sea bottom hills, mountains, or steep slopes may exist. While we rely on a sufficiently up-to-date, high-resolution environmental information, we note that obtaining such information is feasible. In particular, fixed receiver deployments are typically carried out only after having accurately profiled the sea bottom. This makes it possible to choose the location of the receiver such that the surrounding bathymetry is diverse. In addition, the moored receiver can be equipped with cost-effective temperature sensors along its mooring cable, so as to obtain concurrent sound speed estimates.

Our method is based on the modeling of expected acoustic CIRs for different possible locations of the source around the moored receiver. We then assume that the source would emit signals while staying stationary or slowly moving relative to the receiver. Such signals can be the clicks of dolphins, the vocalization of marine mammals, or periodic heartbeatlike pings from an underwater vehicle. We measure the CIR for each received signal, and compare it to the pre-computed modeled CIRs. Thus, we determine the location by the source location of the modeled CIR that best fits the measured one. The result is a sequence of location estimates whose size equals the number of detected emissions. We then create a trellis of possible locations, which are chosen by the output of the cross correlation for the modeled and measured channels and to satisfy an assumed maximum node speed. The final location path of the source is obtained via a process similar to the Viterbi algorithm.

To the best of our knowledge, ours is the only method for the localization of a non-cooperative source from a single reception element. The specific contributions of this paper are:

- A novel approach to estimate the trajectory of a submerged source using a single receiving element;

- A tracking approach to estimate the path of a mobile source in the same conditions.

Our numerical results, which are based on real bathymetry information, show that the proposed approach can estimate the source path with a significantly small error.

The remainder of this paper is organized as follows: Section II provides an account of related work; Section III details the localization algorithm; Section IV presents our performance evaluation; Section V concludes the paper. 


\section{RELATED WORK}

Several approaches for acoustic localization under water have been proposed so far [6], [7]. One of the most common range-based systems is long baseline (LBL), which employs trilateration, and thus requires three or more anchor nodes of known position to be present in the area. Most commercially available LBL systems require round-trip time measurements, and hence the interaction between the mobile node and the anchors. This may pose issues in terms of acoustic bandwidth utilization and energy consumption. LBL systems usually achieve good accuracy, but require a considerable anchor node deployment effort. In comparison, short baseline (SBL) systems can be operated completely from a vessel, either anchored or navigating, by lowering different transponders from different points on the vessel.

If the size of the supporting vessel is exceedingly small and the baseline between transponders becomes too short, ultra-short baseline (USBL) systems are preferred. A USBL device consists of a single array of transponders separated by a distance of up to a few centimeters. The array enables the estimation both of the range and of the angle of arrival (AoA). However the accuracy of AoA estimation is directly dependent on the number of hydrophones and on the array size, which might be an issue for some applications. In many cases, USBL systems are complemented by means to measure the SSP, and thus improve the accuracy of range estimation by accounting for the refractive properties of the underwater channel [8].

The transmission of acoustic signals under water is typically subject to significant multipath distortion. The wealth of multipath arrivals that results can be exploited in passive systems in order to improve the localization accuracy [8], or to localize the source with multiple receivers through an acoustic propagation model [9]. Model-based range-bearing localization with two anchor nodes was introduced and evaluated by [10]. Along the same line, some systems have been proposed that attempt to localize a node based on a collection of acoustic channel fingerprints measured in the localization area [1]. Similarly, [5] proposes to localize based on the matching of a received fingerprint against a set of fingerprints measured by an array of receivers. However, the practical application of systematic fingerprint measurements beyond controlled environments seems limited due to the rapidly changing nature of underwater acoustic channels.

Matched-field processing, a method based on full acoustic field propagation modeling in an underwater waveguide, has also been applied to underwater localization [11]. In this context, it was proposed to leverage the azimuthal asymmetry of the environment [12]. Specifically, a vertical 21-element array is employed to localize a node in $3 \mathrm{D}$, by assuming full knowledge of the environment within the reception range of the array. Matched-field localization is still attracting some interest [13], mainly focused on 2D localization performance improvement.

Unlike in most of the works above, in this paper we propose a system for the localization of a non-collaborative source that is emitting acoustic signals using only a single receiving element. Based on the knowledge of the local bathymetry and SSP, we create a model of multipath propagation in the localization area without any substantial offline preparation phase. Unlike in [5], this process does not require any explicit measurements. We then track the path of the source by leveraging the channel response differences induced by bottom reflections of the acoustic signals from different locations. The above procedure is carried out witho no need for any receiver array.

\section{AlgORITHM DESCRIPTION}

\section{A. Preliminary assumptions and input data}

We consider the problem of localizing an acoustic source that moves in the proximity of a moored receiving element. We assume an underlying algorithm for the detection and estimation the signals emitted by the source. Moreover, we assume that no prior information is available about the location and the instantaneous speed of the source. Our algorithm starts by an initial survey to measure the bathymetry of the area surrounding the moored receiver with a fine resolution. The meter-long resolution obtained by a $400-\mathrm{kHz}$ multibeam sonar is more than sufficient in this respect. We further require periodic direct or indirect measurements of the local SSP.

We assume that the source operates at a given depth range between $z_{\min }^{\mathrm{s}}$ and $z_{\max }^{\mathrm{s}}$, and that it moves at a maximum speed $v_{\max }^{\mathrm{s}}$. We also assume that the maximum distance traveled by the source between two subsequent signal transmissions is known to the receiver. While our method also works without such knowledge, the availability of this information greatly reduces complexity. At different locations indexed by $n=$ $1, \ldots, N_{L}$, the source emits acoustic signals that are detected by the receiver along with all the significant multipath arrivals. The locations are expressed in terms of a cylindrical coordinate system as $\mathbf{x}_{n}^{\mathrm{s}}=\left(u_{n}^{\mathrm{s}}, b_{n}^{\mathrm{s}}, z_{n}^{\mathrm{s}}\right)$ where, at location index $n, u_{n}^{\mathrm{s}} \in$ $\left[0, u_{\max }\right]$ is the great-circle distance in meters between the receiver and the source, $b_{n}^{\mathrm{s}} \in\left[0^{\circ}, 360^{\circ}\right)$ is the bearing of the source with respect to the receiver (i.e., the angle at which the receiver sees the source, measured clockwise from due north) and $z_{n}^{\mathrm{s}} \in\left[z_{\min }^{\mathrm{s}}, z_{\max }^{\mathrm{s}}\right]$. We define the source path as the ordered source location sequence $\left\{\mathbf{x}_{1}^{\mathrm{s}}, \ldots, \mathbf{x}_{N_{L}}^{\mathrm{s}}\right\}$.

As a preliminary step, the receiver sets up a database of modeled CIRs, computed at all points of a cylindric grid designed to span the ranges $\mathcal{U}=\left\{\delta_{u}, 2 \delta_{u}, \ldots, u_{\max }\right\}$, the bearing angles $\mathcal{B}=\left\{\delta_{b}, 2 \delta_{b}, \ldots 360^{\circ}\right\}$, and the depth values $\mathcal{Z}=\left\{z_{\text {min }}^{\mathrm{s}}, z_{\text {min }}^{\mathrm{s}}+\delta z, \ldots, z_{\max }^{\mathrm{s}}\right\}$. The set of grid points is then defined as $\mathcal{G}=\mathcal{U} \times \mathcal{B} \times \mathcal{Z}$, where we denote $\mathrm{g}_{u_{i} b_{i} z_{i}} \in \mathcal{G}$ as the $i$ th grid point, $i=1, \ldots, N_{G}$, where $N_{G}=|\mathcal{G}|$.

\section{B. Source Location Estimation}

For each grid point $\mathbf{g}_{u_{i} b_{i} z_{i}}$, the receiver models the expected CIR using a propagation model. In the present work, we employed the Bellhop ray tracing software [14, Ch. 3], [15] for this purpose. Bellhop provides a list of expected multipath arrivals, along with the complex amplitude, the delay, the reception angle, and the list of bottom and surface reflections 
incurred by each arrival. This information is employed to construct two modeled responses, namely a partial CIR $h_{u_{i} z_{i}}^{(1)}(t)$, with only the specular and surface-reflected arrivals, and the complete CIR $h_{u_{i} b_{i} z_{i}}^{(2)}(t)$. As the specular and surface-reflected arrivals are independent of the bearing of the source relative to the receiver, and rather depend only on the SSP, on $u_{i}$ and on $z_{i}$, the subscript $b_{i}$ has been dropped in $h_{u_{i} z_{i}}^{(1)}(t)$.

From the modeled CIRs, the receiver obtains two separate fingerprints, $h_{u_{i} z_{i}}^{(1)}$ and $h_{u_{i} b_{i} z_{i}}^{(2)}$. When the source is at location $\mathbf{x}_{n}$, its emitted sound is received by the receiver as

$$
r_{n}(t)=h_{u_{n} b_{n} z_{n}}(t) \otimes s(t)+\nu(t),
$$

where $h_{u_{n} b_{n} z_{n}}(t)$ is the actual CIR, $s(t)$ is the signal waveform, $\nu(t)$ is the ambient noise, and $\otimes$ denotes convolution. From $r_{n}(t)$, the receiver evaluates $s(t)$. This can be done using a rake receiver [16], blind source separation [17], or cyclostationary analysis [18], to name a few options. Having $s(t)$, the receiver computes

$$
f_{u_{i} z_{i}}^{(1)}=h_{u_{i} z_{i}}^{(1)} \otimes s(t) \quad \text { and } \quad f_{u_{i} b_{i} z_{i}}^{(2)}=h_{u_{i} b_{i} z_{i}}^{(2)} \otimes s(t) .
$$

The receiver then matches $r_{n}(t)$ against the fingerprints $f_{u_{i} z_{i}}^{(1)}$ and $f_{u_{i} b_{i} z_{i}}^{(2)}$ corresponding to the grid points in $\mathcal{G}$ as follows. First, to limit the complexity of the search, the receiver computes a bound on the depth and range of the source. For example, a bound can be obtained based on a received signal strength indicator (RSSI) as in [19]. In this work, we exploit the modeled CIRs $h_{u_{i} z_{i}}^{(1)}$ to compute, for each point in the grid,

$$
C_{u_{i} z_{i}}^{(1)}(n)=\int_{0}^{+\infty} r_{n}(t) f_{u_{i} z_{i}}^{(1)}(t) \mathrm{d} t
$$

Define $\mathcal{M}^{(1)}(n)$ as the set of all pairs $\left(u_{j}, z_{j}\right)$ corresponding to the peaks of $C_{u_{i} z_{i}}^{(1)}(n)$ that exceed a threshold $\Theta_{D}$, $\forall\left(u_{i}, z_{i}\right) \in \mathcal{G}$. Note that we do not limit set $\mathcal{M}^{(1)}(n)$ to contain just the coordinates of the single grid point that yields the maximum correlation. In fact, in this case the estimation of the correct distance and depth may be hindered by the lack of, e.g., the specular arrival, which in turn could be due to specific bathymetry and SSP patterns. Then, for each $\left(u_{j}, z_{j}\right) \in \mathcal{M}^{(1)}(n)$, and $\forall b \in \mathcal{B}$, we compute the following set of correlations

$$
C_{u_{j} b z_{j}}^{(2)}(n, \tau)=\int_{0}^{+\infty} r_{n}(t) f_{u_{j} b z_{j}}^{(2)}(t+\tau) \mathrm{d} t .
$$

Call $\rho_{\mathbf{x}_{k}}^{n}=\max _{\tau} C_{u_{j} b z_{j}}^{(2)}(n, \tau)$, and define $\mathcal{M}^{(2)}(n)$ as the set of all triples $\mathbf{p}_{k}=\left(u_{k}, b_{k}, z_{k}\right)$ corresponding to the $R^{(2)}$ highest values of $\rho_{\mathbf{p}_{k}}^{n} \forall\left(u_{j}, z_{j}\right) \in \mathcal{M}^{(2)}(n)$ and $\forall b \in \mathcal{B}$, where $R^{(2)}$ is a user-defined parameter.

In ideal conditions, e.g., with an extremely dense grid $\mathcal{G}$, in the absence of noise, and with perfect environmental information, it would be enough to limit set $\mathcal{M}^{(2)}(n)$ to the coordinates of the point $\mathbf{p}_{k}=\left(u_{k}, b_{k}, z_{k}\right)$ for which $\rho_{\mathbf{p}_{k}}^{n}$ is highest. However, it is computationally infeasible to have an exceedingly large set $\mathcal{G}$. Moreover, in any practical scenario, the grid point closest to the actual position of the source might not yield the highest correlation, due to noise, outdated

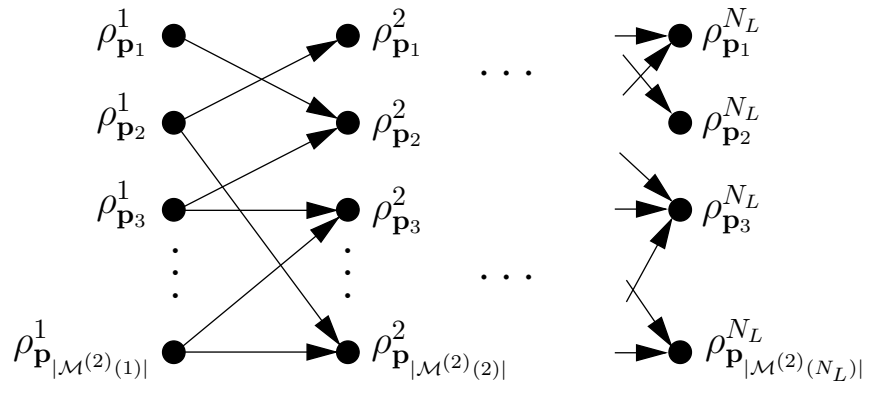

Figure 1. Trellis employed by the path tracking algorithm for source path estimation. Each node represents a location estimate. Trellis links exist only among locations that are closer than the maximum distance covered by the source when traveling at full speed between subsequent signal transmissions.

environmental information, or a combination of both. For these cases, in the following we present a filtering scheme that assumes set $\mathcal{M}^{(2)}(n)$ to contain multiple estimated coordinates.

\section{Source Path Estimation}

Once the receiver has determined $\mathcal{M}^{(2)}(n)$ for $n=$ $1, \ldots, N_{L}$, it proceeds to find the most likely sequence of source locations among all possible options. To that end, we apply a path estimation algorithm, somewhat similar to the Viterbi algorithm. Our algorithm works on a trellis such as the one shown in Fig. 1. Each node represents an estimated location, so that the first column of nodes contains all location estimates for the first detected signal from the source (set $\mathcal{M}^{(2)}(1)$ ), the second column contains the estimates in set $\mathcal{M}^{(2)}(2)$, and the last column those in $\mathcal{M}^{(2)}\left(N_{L}\right)$. We rely on the value of the cross correlation between the modeled and measured channels as a confidence index. In each column $i$, the nodes are sorted in order of decreasing confidence, i.e., $\rho_{\mathbf{p}_{1}}^{i}>\rho_{\mathbf{p}_{2}}^{i}>\cdots>\rho_{\mathbf{p}_{\left|\mathcal{M}^{(2)}(i)\right|}^{i}}^{i}$.

A link exists between a node in column $n$ and a node in column $n+1$ if the corresponding locations are closer than the maximum distance the source could cover when traveling at full speed between the $n$th and the $(n+1)$ th signal detections. Formally, call $e_{\ell_{n} \ell_{n+1}}$ the edge that connects the $\ell_{n}$ th estimated position in column $n$ of the trellis and the $\ell_{n+1}$ th at stage $n+1$. Call $A\left(e_{\ell_{n} \ell_{n+1}}\right)=\mathbf{p}_{\ell_{n}}$ and $S\left(e_{\ell_{n} \ell_{n+1}}\right)=\mathbf{p}_{\ell_{n+1}}$ the ancestor and the successor of edge $e_{\ell_{n} \ell_{n+1}}$, respectively. Define the edge weight as

$$
\sigma\left(e_{\ell_{n} \ell_{n+1}}\right)=\mathbb{1}\left[d\left(\mathbf{p}_{\ell_{n}}, \mathbf{p}_{\ell_{n+1}}\right) \leq v_{\max }^{\mathrm{s}}\left(t_{n+1}-t_{n}\right)\right],
$$

where $\mathbb{1}[\cdot]$ is equal to 1 if the argument is true and 0 otherwise, $d(\mathbf{x}, \mathbf{y})=\|\mathbf{x}-\mathbf{y}\|_{2}$ is the Euclidean distance between locations $\mathbf{x}$ and $\mathbf{y}$, whereas $t_{n}$ and $t_{n+1}$ are the transmission epochs of the $n$th and $(n+1)$ th detected signals, respectively. Edges with zero weight are removed from the trellis. Additionally, if for edge $e_{\ell_{n} \ell_{n+1}}$ it occurs that its ancestor $\mathbf{p}_{\ell_{n}}$ is not successor of any edge $e_{\ell_{n-1} \ell_{n}}$, or that its successor $\mathbf{p}_{\ell_{n+1}}$ is not ancestor of any edge $e_{\ell_{n+1}, \ell_{n+2}}$, the edge $e_{\ell_{n} \ell_{n+1}}$ is removed from the trellis as well.

We remark that a conventional Viterbi approach would include all grid points in $\mathcal{G}$ each column. However, this would 
make the state space very large, and lead to an exceedingly high computational complexity, especially if $|\mathcal{G}|$ is very large. In addition, solving through the Viterbi algorithm would require an estimate for the emission and transition probabilities. Our heuristic version relies on confidence indices, and allows to trim the state space according to physical movement constraints, which in turn reduces the path estimation complexity.

Let $\mathcal{E}(n)=\left\{e_{\ell_{n} \ell_{n+1}}\right\}$ be the set of edges that link a node in column $n$ of the trellis to a node in column $n+1$, and define the following metric for each edge

$$
\lambda\left(e_{\ell_{n} \ell_{n+1}}\right)=\rho_{\mathbf{p}_{\ell_{n}}}^{n} \rho_{\mathbf{p}_{\ell_{n+1}}}^{n+1} \sigma\left(e_{\ell_{n} \ell_{n+1}}\right) .
$$

If for any $n$ it should occur that $\mathcal{E}(n)$ is empty because all edges have been filtered out according to the rule above, or because the transmission has been lost, the trellis would incur a breakage. To correct for these cases, we define edges between nodes in columns $n-1$ and $n+1$, using the rule in (5).

Define a generic path on the trellis as

$$
\Psi=\left\{\hat{e}_{1}, \ldots, \hat{e}_{N_{L}}\right\},
$$

such that $\hat{e}_{1} \in \mathcal{E}(1), \ldots, \hat{e}_{N_{L}} \in \mathcal{E}\left(N_{L}\right)$, and such that $S\left(\hat{e}_{i}\right)=$ $A\left(\hat{e}_{i+1}\right), i=1, \ldots, N_{L}-1$. Define the overall path metric as

$$
\Lambda(\Psi)=\frac{\prod_{i=1}^{N_{L}-1} \lambda\left(\hat{e}_{i}\right)}{\prod_{i=1}^{N_{L}-2} \rho_{S\left(\hat{e}_{i}\right)}^{i}},
$$

i.e., as the product of the confidence metrics for all edges that belong to the path, divided by the confidence of intermediate nodes in order to avoid accounting for them twice. The path estimate is finally found as

$$
\widehat{\Psi}=\underset{\Psi}{\arg \max } \Lambda(\Psi),
$$

where $\widehat{\Psi}=\left\{\hat{\mathbf{x}}_{1}, \hat{\mathbf{x}}_{2}, \ldots, \hat{\mathbf{x}}_{N_{L}}\right\}$. As a means of measuring the discrepancy between the actual and the estimated source paths, we consider the root mean square (RMS) point-wise distance between corresponding points of the two paths. Formally,

$$
\varepsilon_{\widehat{\Psi}}^{d}=\left(\frac{1}{N_{L}} \sum_{n=1}^{N_{L}} d\left(\hat{\mathbf{x}}_{n}, \mathbf{x}_{n}^{\mathrm{s}}\right)^{2}\right)^{1 / 2} .
$$

For several practical applications, it is sufficient to estimate at least the bearing of the source relative to the receiver. We convey the effectiveness of our approach in this respect through the average bearing estimation error, defined as

$$
\varepsilon_{\widehat{\Psi}}^{a}=\frac{1}{N_{L}} \sum_{n=1}^{N_{L}}\left|\hat{b}_{n}-b_{n}^{\mathrm{s}}\right| .
$$

\section{Simulation RESUlts}

\section{A. Scenario and parameters}

For our simulations, we consider a zone in the San Diego bay area, off the coast of US's southern California. We place the receiver at the coordinates $\left[32.9390^{\circ} \mathrm{N}, 117.2816^{\circ} \mathrm{W}\right]$. This choice is inspired from the actual coordinates of a buoy released in the area during a recent sea trial. The hydrophone of the receiver is placed at depth of $10 \mathrm{~m}$. We take a set of accurate bathymetry data from [20] (revealing that the average depth in the area is about $50 \mathrm{~m}$ ), and employ an SSP sample taken at the observed area. The SSP has a downwardrefractive shape, typical of shallow Californian waters during warm seasons. We assume that the water surface is flat. The depth of the source is set to $10 \mathrm{~m}$ in all simulations. Yet, the receiver is unaware of this choice of depth.

The fingerprint grid pre-computed by the receiver spans a total range $u_{\max }=1.5 \mathrm{~km}$ from the receiver (with a resolution of $1 \mathrm{~m}$ ), the whole azimuthal plane (with a resolution of $1^{\circ}$ ), and is computed for all depths between $5 \mathrm{~m}$ and $15 \mathrm{~m}$ (also with a resolution of $1 \mathrm{~m}$ ). Therefore, there are a total of about 6 million points in set $\mathcal{G}$, which would make it impossible to estimate the most likely path through the regular Viterbi algorithm by exploring the whole state space. This motivates the computational complexity reduction efforts described in the previous section. A simulation starts by deploying the emitting source at random in the area at a range of $500 \mathrm{~m}$ from the receiver. The source then chooses a bearing at random and moves along the corresponding direction with constant speed for the time required to carry out 10 transmissions. The locations $\mathbf{x}_{n}^{\mathrm{s}}$ and $\mathbf{x}_{n+1}^{\mathrm{s}}$ where two subsequent emissions take place are such that $d\left(\mathbf{x}_{n}^{\mathrm{s}}, \mathbf{x}_{n+1}^{\mathrm{s}}\right) \leq d_{\max }$, where we set $d_{\max }=50 \mathrm{~m}$.

The signal transmitted by the source, $s(t)$, is chosen to be a linear chirp signal of duration $100 \mathrm{~ms}$ centered at a carrier frequency of $96 \mathrm{kHz}$. This is akin to dolphins clicks. For each emission from a given source-receiver location pairs, the channel impulse response is computed through Bellhop [15], using as parameters the SSP and the available bathymetry samples along the direction from the source to receiver. The ambient noise at the receiver is modeled as an additive white Gaussian process, whose power is tuned so as to achieve a prescribed signal-to-noise ratio (SNR). The received signal is converted to baseband before cross-correlating it with the fingerprints.

For the computation of (3) and the formation of set $\mathcal{M}^{(1)}(n)$, we choose $\Theta_{D}=0.1 \forall n$. A sample result from (3) is shown in Fig. 2a. We observe a clear peak suggesting that the source is located at a distance of approximately $450 \mathrm{~m}$ from the receiver, at a depth of $10 \mathrm{~m}$. This is due to the presence of all expected specular and surface-reflected arrivals in the received signal. If, e.g., the specular arrival were missing, the correlation peak at $500 \mathrm{~m}$ would not be as high. This is why we consider all three significant peaks, including those at about $300 \mathrm{~m}$ and $600 \mathrm{~m}$, and for all depths where such peaks exceed $\Theta_{D}$.

To populate set $\mathcal{M}^{(2)}(n)$, we set $R^{(2)}=70$. A sample computation of (4) for some range-depth pairs in $\mathcal{M}^{(1)}(n)$ is shown in Fig. 2b. While in this particular case a peak stands out corresponding to the correct bearing of about $120^{\circ}$, often such a favorable result does not occur: the chosen value of $R^{(2)}$ allows to considerably increase the probability that the actual bearing is included in $\mathcal{M}^{(2)}(n)$, while keeping the computational effort controlled.

An example of the output of the algorithm for a single step 


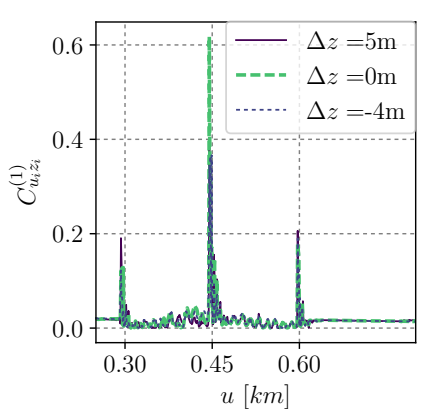

(a) Distance-depth estimation
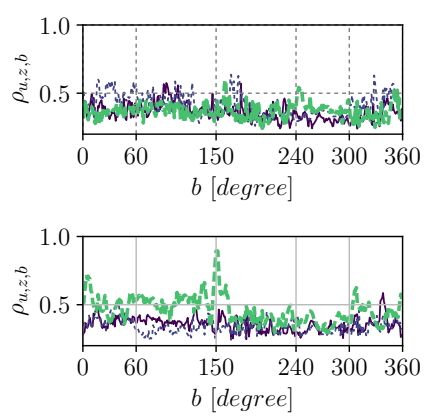

(b) Bearing estimation (top: wrong distance, bottom: correct distance)
Figure 2. Example of correlation values for $\mathbf{x}^{\mathrm{s}}=\left[446 \mathrm{~m}, 150^{\circ}, 10 \mathrm{~m}\right]$ for $\mathrm{SNR}=30 \mathrm{~dB}$.

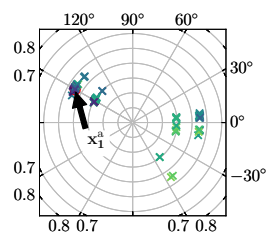

(a) Location estimates after cross-correlation

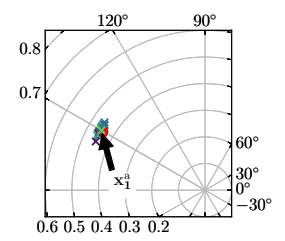

(b) Filtered estimates

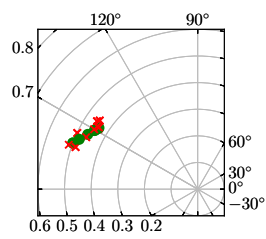

(c) Whole path estimate (red crosses) vs. actual path (green dots)
Figure 3. Example of cross-correlation results for model-based range-bearing localization (distances in $[\mathrm{km}]$ ). Our method successfully rules out spurious locations and achieves an accuracy of $\varepsilon^{d} \approx 160 \mathrm{~m}$ and $\varepsilon^{a} \approx=4.4^{\circ}$.

$n$ and a whole path is provided in Fig. 3. Each panel shows a view of the area around the receiver, which is located at the center of panel (a) or towards the bottom-right corner in panels (b) and (c). Fig. 3a shows the location estimates for the first signal emission, obtained from the set $\mathcal{M}^{(2)}(1)$ as described above; Fig. $3 \mathrm{~b}$ shows the location estimates after filtering out those that are not part of any edge set $\mathcal{E}(n-1)$ or $\mathcal{E}(n), \forall n$; Fig. $3 \mathrm{c}$ shows the final path estimate compared to the actual path of the source. For the numerical results discussed in the following, we consider a Monte-Carlo set of 100 source paths, which corresponds to 1000 signal emissions.

\section{B. Localization accuracy under varying SNR}

We start our performance evaluation by running our algorithm in the presence of exact environmental data under different SNR values. The complementary cumulative distribution functions (CCDF) of the distance RMS error (RMSE) and of the bearing error are shown in Figs. $4 \mathrm{a}$ and $4 \mathrm{~b}$, respectively. Thanks to the perfect knowledge of both the bathymetry and the SSP in the observed area, neither result shows a significant dependence on the SNR, even after decreasing it to as low as $3 \mathrm{~dB}$, which in turn tends to make additional peaks appear in the correlation outputs. We observe that the average RMSE varies from about $120 \mathrm{~m}$ for an SNR of $30 \mathrm{~dB}$, up to about $170 \mathrm{~m}$ for an SNR of $3 \mathrm{~dB}$, with a median error around $80 \mathrm{~m}$ which is remarkably good given the grid resolution employed and the use of a single receiving element. The bearing estimation results show even higher accuracy, with

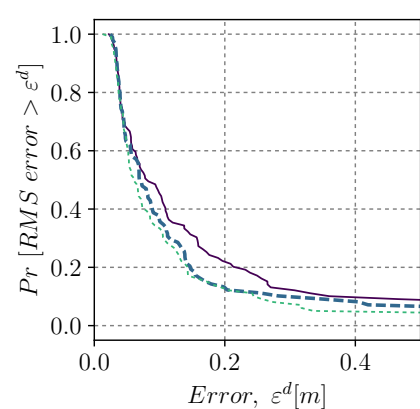

(a) Distance RMSE CCDF

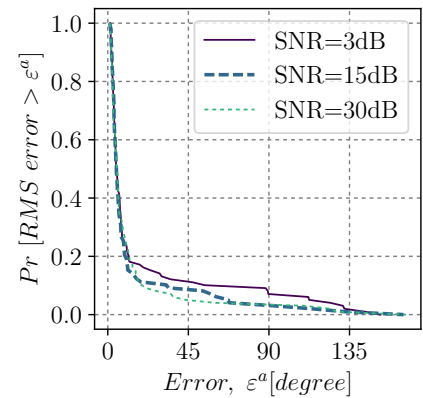

(b) Bearing error $\mathrm{CCDF}$
Figure 4. Accuracy of the path estimation algorithm in the presence of exact environmental data, for different values of the SNR. Even for low values of the SNR the arrival structure does not change considerably, and has no significant effects on the accuracy of our method.

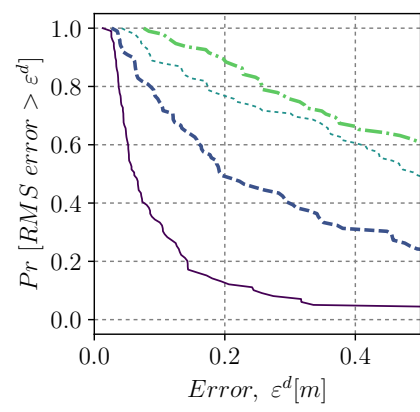

(a) Distance RMSE CCDF

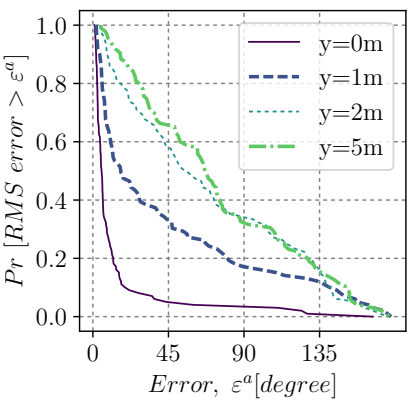

(b) Bearing error $\mathrm{CCDF}$
Figure 5. Accuracy of the path estimation algorithm in the presence of imperfect bathymetry data. Erroneous bathymetry significantly affects the algorithm performance. For limited errors $(y=1 \mathrm{~m})$ the results are still practical for several applications.

a mean estimation error $\varepsilon_{a}<20^{\circ}$ even for an SNR of $3 \mathrm{~dB}$, and a median error of less than $10^{\circ}$.

\section{Localization accuracy under imperfect bathymetry data}

We now proceed by evaluating the sensitivity of the algorithm to imperfect environmental information, starting with bathymetry data. Specifically, we assume that the bathymetry samples available to the receiver are offset with respect to the true samples by an error drawn uniformly at random in the interval $[-y, y]$, where $y$ (in $\mathrm{m}$ ) is a tunable parameter. For each erroneous bathymetry draw, we consider a Monte-Carlo set of 100 source paths and show the CCDFs of the distance RMSE and of the bearing estimation errors in Figs. 5a and 5b, respectively. We observe that wrong bathymetry data decreases the path estimation performance, as expected. However, for a limited offset on bathymetry samples, up $\pm 1 \mathrm{~m}$, the median RMS distance error remains below $200 \mathrm{~m}$, which is still a reasonably good result given the presence of a single receiving element. Instead, an error of up to $\pm 5 \mathrm{~m}$ yields significantly worse performance. However, this is an extreme case, as such an error amounts to about $10 \%$ of the average sea bottom depth in the area, and thus causes significant changes in the structure of the arrivals observed by the receiver. 


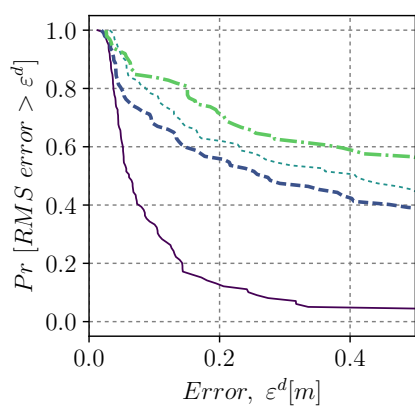

(a) Distance RMSE CCDF

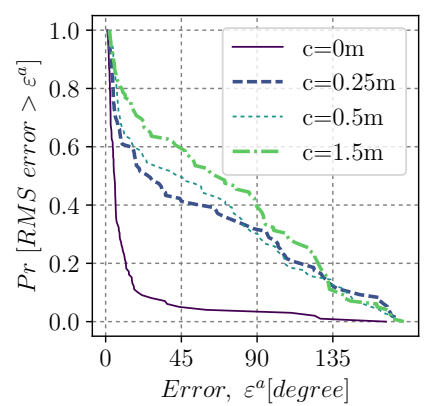

(b) Bearing error CCDF
Figure 6. Accuracy of the path estimation algorithm in the presence of imperfect SSP data. An inaccurate or outdated SSP significantly changes the multipath arrival structure. Still, for $c=0.25 \mathrm{~m}$ the achieved accuracy still has practical utility.

Similar conclusions can be drawn for the bearing estimation error. Fig. 5b shows that the for $y=1 \mathrm{~m}$, the average and median error are about $45^{\circ}$ and $20^{\circ}$, respectively, and increase to respectively $75^{\circ}$ and $55^{\circ}$ for $y=5 \mathrm{~m}$. Still, we observe that even such rough estimates can be instrumental for several applications, including security and environmental monitoring systems (where a rough estimate can trigger a more accurate investigation by human personnel or more complex autonomous vehicles) or fauna and habitat monitoring applications (where it is often sufficient to find the approximate path followed by a vocalizing animal).

\section{Localization accuracy under imperfect SSP data}

We conclude by evaluating the localization accuracy in the presence of outdated SSP data. Specifically, we add an offset drawn uniformly at random in the interval $[-c, c]$ to each SSP sample available to the receiver. While this offset tends to roughly preserve the general refractive properties of the simulated medium (i.e., the general behavior remains downward-refractive), even a small value of $c$ tends to induce significant changes in the trajectory and delay of different arrivals. The simulations confirm this, as for $c=0.25 \mathrm{~m}$ we already observe worse RMS distance error performance (Fig. 6a), with mean and median errors respectively equal to about $400 \mathrm{~m}$ and $250 \mathrm{~m}$. As expected, increasing $c$ tends to reduce the accuracy. The bearing estimation results confirm this trend, showing similar average performance for different values of $c$, although the median error is limited to about $25^{\circ}$ for $c=0.25 \mathrm{~m}$. This results emphasizes the need for periodic SSP measurements performed at the moored receiver.

\section{CONCLUSIONS AND FUTURE WORK}

We presented a novel approach for anchorless localization and path estimations of a non-cooperating underwater source emitting acoustic signals. Our approach relies on a single receiver element and on the erratic use of acoustic propagation in shallow waters. Our method is based on the comparison of the channel estimate taken from a received acoustic signal against a database of channel fingerprints. The latter are modeled instead of measured, so that no periodic channel fingerprint acquisition in the area around the receiver is required. Locations that show a good match between the measured and modeled channels are setup in a lattice form while limiting transitions according to an assumed maximal velocity for the source. The location path is then estimated as the path that yields the best overall cross-correlation. Our approach makes it possible to estimate the path traveled by the source with remarkably good accuracy, given the presence of a single receiver, and with low complexity. Such accuracy decreases (but still remains sufficient for a variety of applications) if the receiver obtains outdated environmental data.

\section{REFERENCES}

[1] V. Chandrasekhar et al., "Localization in underwater sensor networks: survey and challenges," in Proc. ACM WUWNet, 2006, pp. 33-40.

[2] J. J. Leonard and A. Bahr, "Autonomous underwater vehicle navigation," in Handbook of Ocean Engineering. Springer, 2016, pp. 341-358.

[3] Z. Zhou et al., "Scalable localization with mobility prediction for underwater sensor networks," IEEE Trans. Mobile Comput., vol. 10, no. 3, pp. 335-348, 2011.

[4] D. Mellinger et al., "An overview of fixed passive acoustic observation methods for cetaceans," Oceanography, vol. 20, no. 4, pp. 36-45, 2007.

[5] K.-C. Lee et al., "A novel location estimation based on pattern matching algorithm in underwater environments," Elsevier Applied Acoustics, vol. 70, no. 3, pp. 479-483, 2009.

[6] H.-P. Tan et al., "A survey of techniques and challenges in underwater localization," Elsevier Ocean Engineering, vol. 38, no. 14, pp. 1663 1676, 2011.

[7] L. Paull et al., "AUV navigation and localization: A review," IEEE J. Ocean. Eng., vol. 39, no. 1, pp. 131-149, 2014.

[8] E. Dubrovinskaya, I. Nissen, and P. Casari, "On the accuracy of passive multipath-aided underwater range estimation," in Proc. UComms, Lerici, Italy, 2016.

[9] C. O. Tiemann, M. B. Porter, and L. N. Frazer, "Localization of marine mammals near Hawaii using an acoustic propagation model," J. Acoust. Soc. Am., vol. 115, no. 6, pp. 2834-2843, 2004.

[10] J. Gebbie, M. Siderius, and J. S. Allen III, "A two-hydrophone range and bearing localization algorithm with performance analysis," J. Acoust. Soc. of America, vol. 137, no. 3, pp. 1586-1597, 2015.

[11] A. B. Baggeroer, W. A. Kuperman, and P. N. Mikhalevsky, "An overview of matched field methods in ocean acoustics," IEEE J. Ocean. Eng. vol. 18, no. 4, pp. 401-424, 1993.

[12] J. S. Perkins and W. Kuperman, "Environmental signal processing: Three-dimensional matched-field processing with a vertical array," J. Acoust. Soc. of America, vol. 87, no. 4, pp. 1553-1556, 1990.

[13] K. L. Gemba, W. S. Hodgkiss, and P. Gerstoft, "Adaptive and compressive matched field processing," J. Acoust. Soc. of America, vol. 141, no. 1, pp. 92-103, 2017.

[14] F. Jensen et al., Computational Ocean Acoustics, 2nd ed. New York: Springer-Verlag, 1984.

[15] M. Porter et al., "Bellhop code," Last time accessed: June 2017. [Online]. Available: http://oalib.hlsresearch.com/Rays/index.html

[16] L. Marchetti and R. Reggiannini, "An efficient receiver structure for sweep-spread-carrier underwater acoustic links," IEEE Journal of Oceanic Engineering, vol. 41, no. 2, pp. 440-449, 2016.

[17] C. Boya et al., "Identification of multiple partial discharge sources using acoustic emission technique and blind source separation," IEEE Trans. Dielectr. Electr. Insul., vol. 22, no. 3, pp. 1663-1673, Jun. 2015.

[18] S. Byun et al., "Cyclostationary analysis of underwater noise for vehicle propeller monitoring," in Proc. MTS/IEEE OCEANS, Monterey, CA, Sep. 2016.

[19] R. Diamant, H.-P. Tan, and L. Lampe, "LOS and NLOS classification for underwater acoustic localization,” IEEE Trans. Mobile Comput., vol. 13, no. 2, pp. 311-323, 2014.

[20] D. Divins and D. Metzger. (2016) US coastal relief model. [Online]. Available: http://www.ngdc.noaa.gov/mgg/coastal/coastal.html 